\title{
RoboGen: Robot Generation through Artificial Evolution
}

\author{
Joshua E. Auerbach, Deniz Aydin, Andrea Maesani, Przemyslaw M. Kornatowski, \\ Titus Cieslewski, Grégoire Heitz, Pradeep R. Fernando, Ilya Loshchilov, Ludovic Daler and Dario Floreano \\ Laboratory of Intelligent Systems \\ Ecole Polytéchnique Fédérale de Lausanne \\ joshua.auerbach@epfl.ch
}

\section{Extended Abstract}

Science instructors from a wide range of disciplines agree that hands-on laboratory components of courses are pedagogically necessary (Freedman, 1997). However, certain shortcomings of current laboratory exercises have been pointed out by several authors (Mataric, 2004; Hofstein and Lunetta, 2004). The overarching theme of these analyses is that hands-on components of courses tend to be formulaic, closed-ended, and at times outdated. To address these issues, we envision a novel platform that is not only a didactic tool but is also an experimental testbed for users to play with different ideas in evolutionary robotics (Nolfi and Floreano, 2000), neural networks, physical simulation, 3D printing, mechanical assembly, and embedded processing.

Here, we introduce RoboGen ${ }^{\mathrm{TM}}$ : an open-source software and hardware platform designed for the joint evolution of robot morphologies and controllers a la Sims (1994); Lipson and Pollack (2000); Bongard and Pfeifer (2003). RoboGen has been designed specifically to allow evolved robots to be easily manufactured via widely available desktop 3Dprinters ${ }^{1}$, and the use of simple, open-source, low-cost, offthe-shelf electronic components. RoboGen features an evolution engine complete with a physics simulator, as well as utilities both for generating design files of body components for 3D printing, and for compiling neural-network controllers to run on an Arduino microcontroller board ${ }^{2}$.

In this paper, we describe the RoboGen platform, and provide some metrics to assess the success of using it as the hands-on component of a masters-level bio-inspired artificial intelligence course.

\section{Software Suite}

The RoboGen software suite is comprised of two main components: an evolution engine that generates and reproduces robots, and a simulator that renders the evolutionary environment and assesses the fitness of the evolved solutions. Users may go from serial fitness evaluations (using a single

\footnotetext{
${ }^{1}$ Such as the MakerBot Replicator 2x: http: //store.makerbot.com/replicator $2 x$

${ }^{2}$ http: //www. arduino.cc
}
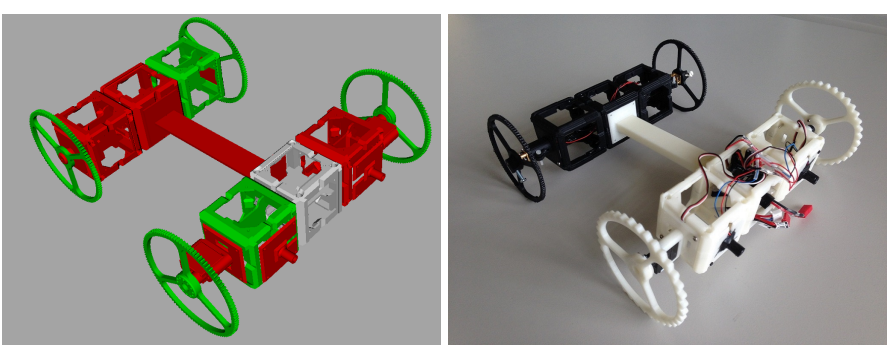

Figure 1: Sample robot evolved with RoboGen: simulation (left) and reality (right).

simulator) to massive parallelism distributed across a network depending on their computational resources.

Robot bodies Robots evolved with RoboGen (see Fig. 1) are composed of predefined and parameterized modules, and are represented as genetic programming trees (Koza, 1992). The modular building blocks that make up the body representations include passive and active structural elements as well as sensing components. A full list of components, and their detailed specifications may be found on the RoboGen website http: / / www. robogen. org.

Robot brains The "brains" of the RoboGen robots are fully-connected, recurrent artificial neural networks. The robots can sense their environment through touch sensors, light sensors and a six degree-of-freedom inertial measurement unit (IMU). The number of sensors and actuators used in robots increase the complexity of the simulations, but their use may be necessary to evolve robots that are truly adapted to diverse tasks and environments.

In the classroom environment, we provide several scenarios for the students to explore the utility of various parameters and components of the software suite. Specifically, we aim to promote an understanding of how the tasks and environments affect the evolved morphologies (Auerbach and Bongard, 2014), and how allowed simulation complexity changes the adaptedness of the robots generated through the evolutionary process. 
The complexity of simulations achievable with this software is entirely dependent on the user: beginners can familiarize themselves with new concepts in a more controlled way by evolving only the neural network controllers, whereas advanced users can work on the evolution engine to customize the evolutionary algorithm or simulator or even introduce new morphological building blocks. This openendedness is a major advantage of the platform and addresses the current concerns regarding science laboratory education (Mataric, 2004). Additionally, our software is also the first educational platform that provides the users with the ability to manufacture their own evolved robots. By allowing users to get completely immersed in the artificial evolution process, we hope to encourage users to think about real-life applicability of their simulations. Finally, we aim to foster collaborations among groups of students with different expertise by having them design evolutionary scenarios, carry out experiments, and test their evolved designs in hardware.

\section{Teaching Assessment}

Discerning whether RoboGen is indeed an effective tool for teaching evolutionary robotics requires an analytical approach. For this reason, we devised a measuring tool to get a sense of how well the students in our class meet the desired learning outcomes. In our teaching assessment we focus on a set of measurable learning outcomes that were defined based on the "Content, Skills and Values (CSV)" classification of learning outcomes (Carleton University, 2014). A brief questionnaire was prepared to be administered twice during the course: once after the first in-depth introduction to the RoboGen project (but before the students begin working on the project), and once at the end of the project. The purpose of this scheduling is to determine the improvement in the technical skills targeted in this course.

The evaluation questions fall under one of the aforementioned CSV categories, and are answered on a Likert scale from 1 to 5 , with 5 being the strongest positive response (Likert, 1932). The first questionnaire saw $67 \%$ participation (53 students).

The psychosocial and environmental factors that influence learning will be measured through a separate questionnaire administered at the end of the course, determining both the students' perception of the classroom environment for learning and their "ideal" environment. This questionnaire is an adapted version of the Science Laboratory Environment Inventory tailored to suit the teaching environment of the course given at EPFL (Fraser et al., 1995).

\section{Conclusions}

Overall, we envision RoboGen as not only an effective platform for evolving the morphologies and controllers of manufacturable robots, but also a valuable educational tool. It is a system that should be attractive to researchers, hobby- ists, educators and students alike. Going forward we hope to develop a worldwide community around RoboGen. Users will be able to discuss their experiences, share ideas, and contribute to the growth of the project by introducing new morphological building blocks, evolutionary scenarios, and educational exercises.

\section{Acknowledgements}

We acknowledge the contributions of the students of MICRO-551 at EPFL, and the members of LIS.

The research leading to these results has received funding partially from the European Union Seventh Framework Programme (FP7/2007-2013) under grant agreement $\mathrm{n}^{\circ} 308943$.

\section{References}

Auerbach, J. E. and Bongard, J. C. (2014). Environmental influence on the evolution of morphological complexity in machines. PLoS computational biology, 10(1):e1003399.

Bongard, J. C. and Pfeifer, R. (2003). Evolving complete agents using artificial ontogeny. In Morpho-functional Machines: The New Species (Designing Embodied Intelligence, pages 237-258. Springer-Verlag.

Carleton University (2014). Writing learning outcomes. http://carleton.ca/edc/wp-content/ uploads/Writing-Learning-Outcomes.pdf.

Fraser, B. J., Giddings, G. J., and McRobbie, C. J. (1995). Evolution and validation of a personal form of an instrument for assessing science laboratory classroom environments. Journal of Research in Science Teaching, 32(4):399-422.

Freedman, M. P. (1997). Relationship among laboratory instruction, attitude toward science, and achievement in science knowledge. Journal of Research in Science Teaching, 34(4):343-357.

Hofstein, A. and Lunetta, V. N. (2004). The laboratory in science education: Foundations for the twenty-first century. Science Education, 88(1):28-54.

Koza, J. (1992). Genetic Programming: On the Programming of Computers by Means of Natural Selection. MIT Press, Boston, MA.

Likert, R. (1932). A technique for the measurement of attitudes. Archives of Psychology, 22 140:55.

Lipson, H. and Pollack, J. (2000). Automatic design and manufacture of robotic lifeforms. Nature, 406:974-978.

Mataric, M. J. (Spring 2004). Robotics education for all ages. In Proceedings, AAAI Spring Symposium on Accessible, Handson AI and Robotics Education.

Nolfi, S. and Floreano, D. (2000). Evolutionary Robotics: The Biology,Intelligence, and Technology. MIT Press, Cambridge, MA, USA.

Sims, K. (1994). Evolving virtual creatures. In SIGGRAPH '94: Proceedings of the 21 st annual conference on Computer graphics and interactive techniques, pages 15-22, New York, NY, USA. ACM. 